\title{
Identification and characterization of two CD4 alleles in Microminipigs
}

\author{
Tatsuya Matsubara ${ }^{1,2}$, Naohito Nishii ${ }^{1,2}$, Satoshi Takashima², Masaki Takasu ${ }^{1,2}$, Noriaki Imaeda², Kayo Aiki-Oshimo², \\ Kazuaki Yamazoe ${ }^{1,2}$, Michinori Kakisaka ${ }^{3}$, Shin-nosuke Takeshima ${ }^{3}$, Yoko Aida ${ }^{3}$, Yoshie Kametani ${ }^{4}$, Jerzy K. Kulski ${ }^{4,5}$, \\ Asako Ando ${ }^{4}$ and Hitoshi Kitagawa ${ }^{1,2}$
}

\begin{abstract}
Background: We previously identified two phenotypes of CD4+ cells with and without reactions to anti-pig CD4 monoclonal antibodies by flow cytometry in a herd of Microminipigs. In this study, we analyzed the coding sequences of CD4 and certified the expression of CD4 molecules in order to identify the genetic sequence variants responsible for the positive and negative PBMCs reactivity to anti-pig CD4 monoclonal antibodies.

Results: We identified two CD4 alleles, CD4.A and CD4.B, corresponding to antibody positive and negative, respectively, by nucleotide sequencing of $P C R$ products using CD4 specific primer pairs. In comparison with the swine CD4 amino-acid sequence [GenBank: NP_001001908], CD4.A had seven amino-acid substitutions and CD4.B had 15 amino-acid substitutions. The amino-acid sequences within domain 1 of CD4.B were identical to the swine CD4.2 [GenBank: CAA46584] sequence that had been reported previously to be a modified CD4 molecule that had lost reactivity with an anti-pig CD4 antibody in NIH miniature pigs. Homozygous and heterozygous CD4.A and CD4.B alleles in the Microminipigs herd were characterised by using the RFLP technique with the restriction endonuclease, BseRl. The anti-pig CD4 antibody recognized pig PBMCs with CD4.AA and CD4.AB, but did not recognized those with CD4. $B B$. We transfected HeLa cells with the FLAG-tagged CD4.A or CD4.B vectors, and certified that transfected HeLa cells expressed FLAG in both vectors. The failure of cells to react with anti-CD4 antibodies in CD4.B pigs was associated to ten amino-acid substitutions in domain 1 and/or one amino-acid substitution in joining region 3 of CD4.B. We also found exon 8 was defective in some CD4.A and CD4.B resulting in the loss of the transmembrane domain, which implies that these CD4 proteins are secreted from helper T cells into the circulation.
\end{abstract}

Conclusions: We identified that amino-acids substitutions of domain 1 in CD4.B gave rise to the failure of some CD4 expressing cells to react with particular anti-pig CD4 monoclonal antibodies. In addition, we developed a PCR-RFLP method that enabled us to simply identify the CD4 sequence variant and the positive and negative PBMCs reactivity to our anti-pig CD4 monoclonal antibodies without the need to use flow cytometric analysis.

Keywords: CD4 polymorphism, PCR-RFLP, Amino-acid substitution, Microminipigs

\section{Background}

The CD4 molecule is a cell-surface glycoprotein receptor expressed by helper $\mathrm{T}$ cells, monocytes, macrophages, and dendritic cells; and its structure consists of four immunoglobulin-like domains (D 1 to D 4) as part of the extracellular domain, a transmembrane domain, and

\footnotetext{
* Correspondence: nishii@gifu-u.ac.jp

1 United Graduate School of Veterinary Sciences, Gifu University, Gifu 501-1193, Japan

2Department of Veterinary Medicine, Faculty of Applied Biological Sciences, Gifu University, 1-1 Yanagido, Gifu 501-1193, Japan

Full list of author information is available at the end of the article
}

a cytoplasmic tail $[1,2]$. The extracellular domain binds to the monomorphic region of MHC class II to increase the affinity of the $\mathrm{T}$ cell receptor to the antigen peptideMHC class II complex [3, 4]. The cytoplasmic portion of CD4+ recruits tyrosine kinase, Lck, and the kinase enhances signal transduction in T cell activation $[5,6]$.

Microminipigs are extra-small and novel miniature pigs developed for biomedical research in Japan [7]. Recently, swine leukocyte antigen (SLA) haplotypes were assigned in a herd of Microminipigs in order to further investigate their immunological characteristics [8] during 
disease and infections. In the process of analyzing helper $\mathrm{T}$ cell function, we found that some pigs had CD4+ cells that could not be detected by flow cytometry while using three anti-pig CD4 monoclonal antibodies of the clones 74-12-4, MIL17, and PT90A [9]. The pedigree analysis indicated that the CD4-undetectable trait might be recessive, suggesting gene variation [9]. Failure of CD4 cells to react with an anti-pig CD4 antibody was reported previously in the $\mathrm{NIH}$ miniature swine [10] and the presence of partial nucleotide sequences and 10 amino-acid substitutions in exon 3 and 4 of two kinds of CD4 alleles $(C D 4.1, C D 4.2)$ in these miniature swine might be the cause of helper $\mathrm{T}$ cells not reacting with the anti-pig CD4 antibody [11]. On the other hand, because Microminipigs have no consanguinity with $\mathrm{NIH}$ miniature swine, the cause for the failure of CD4 cells to react with the anti-pig CD4 antibody in Microminipigs might be different from NIH miniature swine. Hence, we need to clarify the variations in the CD4 nucleotide and amino acid sequences for the positive and negative antibody phenotypes in Microminipigs.

In this study, in order to clarify the reasons for the failure of the anti-pig CD4 antibodies to react with and detect peripheral CD4+ cells and to assess whether sequence variations within the CD4 molecules of Microminipigs might cause immunological alterations, we 1) sequenced and analyzed the coding sequence (CDS) of CD4 using genomic DNA and reverse transcribed (RT)PCR products of CD4 mRNA in Microminipigs,, 2) developed a simple PCR-RFLP method to identify the CD4 sequence variant and the positive and negative PBMCs reactivity to anti-pig CD4 monoclonal antibodies, and 3) examined the expression of the CD4 alleles transfected into HeLa cells.

\section{Methods}

\section{Microminipigs}

Microminipigs were raised in a conventional environment at Fuji Micra Inc. (Fujinomiya, Japan) or Gifu
University. This study was carried out along the Gifu University Laboratory Animal Guidelines.

\section{Flow cytometric analysis}

Flow cytometry was performed as previously [9]. Briefly, peripheral blood mononuclear cells (PBMCs) of 231 Microminpigs were isolated using Lymphoprep (Axis Shield, Oslo, Norway), stained with a FITC-conjugated anti-pig CD4a antibody (clone 74-12-4, BD Biosciences, San Jose, CA), and analyzed using FACSCalibur (BD Biosciences) to classify the pigs with and without CD4 affinity for the 74-12-4 antibody. The data was analyzed with FlowJo version 7.6.5 software (FlowJo, Ashland, OR). The antibody reactivity with the CD4 protein was measured as the MFI (median of fluorescence intensity) of CD4+ cells in PBMCs.

\section{Direct sequencing of CD4 coding region}

Genomic DNA was extracted from peripheral blood, pieces of tail or ear tissues of 11 Microminipigs (reactive CD4: six pigs, non-reactive CD4: five pigs) using a Wizard Genomic DNA Purification Kit (Promega Corporation, Madison, WI). CD4 gene-specific primer pairs for amplification and sequencing of the coding region (exons 2 to 10) were designed as shown in Table 1 based on a swine reference sequence [Genbank: NC_010447]. The PCR cycling parameters included an initial denaturation step of $4 \mathrm{~min}$ at $94{ }^{\circ} \mathrm{C}, 35$ cycles consisting of $30 \mathrm{~s}$ at $94{ }^{\circ} \mathrm{C}$, $30 \mathrm{~s}$ at $60{ }^{\circ} \mathrm{C}, 30 \mathrm{~s}$ at $72{ }^{\circ} \mathrm{C}$, and a final extension step of $7 \mathrm{~min}$ at $72{ }^{\circ} \mathrm{C}$. Sequencing was performed with ABI PRISM 3100 (Life Technologies, Carlsbad, CA) using Big Dye Terminator ver. 3.1 (Life Technologies). The nucleotide and deduced amino-acid sequence results were aligned using GENETYX version 10 (GENETYX, Tokyo, Japan) with three swine CD4 sequences: [GenBank: NM_001001908, GenBank: X65629 (CD4.1), GenBank: X65630 (CD4.2)].

Table 1 CD4 exonic primer sets for DNA sequencing

\begin{tabular}{llll}
\hline Amplification exon & Forward primer sequence & Reverse primer sequence & Length of PCR product size (bp) \\
\hline 2 & 5'-GTACCTGTGGGTGTCAGTTAGAG-3' & 5' -CTTACCCAGCACCAGATATTTTC-3' & 383 \\
3 & 5'-CTCAGACTCAAACTGGGATGATTG -3' & 5' -GATCCCAGAGTTAATAGGAGCTG-3' & 366 \\
4 & 5'-AATGAGCAACTCAGATCAGAAGAGT-3' & 5'-CTTATCCATCTCTGGACGGTG-3' & 363 \\
5 & 5'-CTTCTCCTTGGGGATAGTGCAT-3' & 5'-ACACTACAGCCACGAGCAGAG-3' & 358 \\
6 & 5'-GCCTAGAGCTAGATGGGAATTAAG -3' & 5'-GATTCCAGCCTCAGTTCAAACC-3' & 617 \\
7 & 5'-CTTAGAGCAGACAAGTGCTAGGAA- 3' & 5'-ACCATACCCATAACCCACTGACTC-3' & 373 \\
8 & 5'-AGCATAAGGATCAGACCCAAGTGT-3' & 5'-TAACTCTGTGGCTTCTTGTCTCTC-3' & 400 \\
9 & 5'-GTTAATTCTGGGACAGATGGCTTC-3' & 5'-CTCTCTTCACCCCTCCTCTTTG-3' & 238 \\
$10-1$ & 5'-CCATCTCTGTGCAGGAAAAGTC-3' & 5'-AGCTGAGCTGCTTGGGTGATA-3' & 698 \\
$10-2$ & 5'-ACTGACGGAGCCACAGACTC-3' & 5'-GGCTATCAACTTTCGCAGGA-3' & 668 \\
\hline
\end{tabular}




\section{CD4 genotyping by PCR-restriction fragment length polymorphism (PCR-RFLP) method}

The PCR-RFLP technique in association with the restriction enzyme BseRI was used to identify and differentiate between the two CD4 alleles. PCR amplification was performed on genomic DNA to amplify CD4 exon 3, and the PCR products were digested with an enzyme, BseRI (New England Biolabs Inc., Ipswich, MA). The allele-specific bands were analyzed by $2 \%$ agarose gel electrophoresis.

We determined the hereditary pattern of CD4 alleles of sibs from heterozygous parents by using the PCRRFLP method to genotype 64 piglets, 35 males and 29 females, born from 17 matings. In addition, CD4 genotypes and phenotypes were assigned in 143 Microminipigs by the PCR-RFLP methods and flow cytometry as described above. The percentage and MFI of CD4+ cells in PBMCs were also compared between the two CD4 genotypes.

\section{Detection of CD4 mRNA by analyzing RT-PCR products} Peripheral blood samples of Microminipigs were collected into Paxgene Blood RNA tubes (PreAnalytiX, Hombrechtikon, Switzerland). Total RNA was extracted using a PAXgene Blood RNA Kit (PreAnalytiX). Complementary DNA (cDNA) was synthesized from oligo dT primers using total RNA and the reverse transcriptase kit, ReverTra Ace (TOYOBO, Osaka, Japan).

To characterise the expressed CD4 mRNA, RT-PCR amplification was performed between exon 1 and 4 or exon 1 and 5 by using two specific primer pairs (Table 2) that were designed from the nucleotide sequence information of two CD4 alleles, CD4.A and CD4.B, obtained from Microminpigs and the swine CD4 reference sequence [GenBank: NM_001001908]. RT-PCR was performed using the same conditions as those used for sequencing amplification. The RT-PCR products were digested with BseRI, and electrophoresed in $2 \%$ agarose gel.

\section{Transfection of two kinds of CD4 alleles into HeLa cells}

We chose HeLa cells for the analysis of CD4 expression analysis in Microminipigs because the same cells have been used for the analysis in human [12, 13]. To verify the differences in the antibody reactivity of CD4 alleles, HeLa cells were transfected with the FLAG-tagged CD4 vectors (CD4-FLAG) constructed by adding FLAG to C-

Table 2 Primers for RT-PCR to detect the expression of CD4 mRNA

\begin{tabular}{llll}
\hline Name & $\begin{array}{l}\text { Forward primer } \\
\text { sequence }\end{array}$ & $\begin{array}{l}\text { Reverse primer } \\
\text { sequence }\end{array}$ & $\begin{array}{l}\text { Length of PCR } \\
\text { products (bp) }\end{array}$ \\
\hline Primer 1 & $\begin{array}{l}\text { 5'-GTAAGAGAAGCA } \\
\text { GAGGGGAAGAG-3' }\end{array}$ & $\begin{array}{l}\text { 5'-GATTCTTGATGA } \\
\text { TCAGGGGAAAG-3' }\end{array}$ & 400 \\
Primer 2 & 5'-GTAAGAGAAGCA $^{\prime}$ '-CATTCTTGCTTT & 595 \\
& GAGGGGAAGAG-3' & ATTCCCTGGAC-3' & \\
\hline
\end{tabular}

terminus of the two different CD4 alleles. PBMCs were isolated from two pigs genotyped to two types of CD4 homozygotes using the Lymphoprep kit (Axis Shield) and their total RNAs were extracted with ISOGEN (NIPPON GENE, Tokyo, Japan). cDNA was synthesized by SuperScript III First-Strand Synthesis System (Life Technologies) using a sequence specific reverse primer (5'TCAGGTGAGGGAATAGTTCTTCTGTTGCCG-3'). R T-PCR was performed with PrimeSTAR Max DNA Polymerase (TaKaRa Bio Inc., Otsu, Japan) and a CD4 specific primer pair containing $X h o I$ and Not recognition sequences and FLAG sequences (Forward: 5' -TATCTCGAGATGGACCCAGGAACCTCTCT-3'; Reverse: 5' - T ATGCGGCCGCTCACTTGTCATCGTCCTTGTAATC GGTGAGGGAATAGTTCTTCTTCTGTTGC-3'). The RT-PCR products of CD4-FLAG were cloned into the mammalian expression vector pCAGGS [14], and the integrity of the constructed vectors were confirmed by DNA sequencing using the following five primers; Forward 1: 5'-GCAGGGACTTCCTTTGTCCCAAAT-3'; Forward 2: 5' - TATCTCGAGATGGACCCAGGAACCT CTCT-3'; Forward 3: 5'-AGTCACCCTACAGTGCA ATGGAAAG-3'; Reverse 1: 5' - TATGCGGCCGCTCA CTTGTCATCGTCCTTGTAATCGGTGAGGGAATAG TTCTTCTTCTGTTGC-3'; Reverse 2: 5'-TGTCCTTC CGAGTGAGAGACACAA-3'. The constructed plasmids were transfected into HeLa cells using Lipofectamine 2000 (Life Technologies) according to the manufacturer's instructions. After culturing for $20 \mathrm{~h}$, the transfected HeLa cells were stained with a rabbit anti-FLAG polyclonal antibody (Sigma-Aldrich, St. Louis, MO) followed by the Alexa Fluor 488 goat anti-rabbit IgG antibody (Life Technologies). The PE-conjugated mouse anti-pig CD4a monoclonal antibody (clone 74-12-4, SouthernBiotech, Birmingham, UK) was used for the CD4 molecule detection. The cells were also stained with Hoechst 33342 (ImmunoChemistry Technologies, Bloomington, IN). Fluorescence imaging was conducted using scanning laser confocal microscope FV1000-D IX81 (Olympus, Tokyo, Japan).

\section{Statistical analysis}

In the hereditary analysis, the observed and theoretical values were assessed by the chi-squared test using Excel 2007 (Microsoft, Seattle, WA) with an add-in software Statcel 3 (OMS, Tokorozawa, Japan). Theoretical values were determined on the basis of the Punnett square. A difference of $P<0.05$ was considered as significant.

\section{Results}

PCR amplification of CD4 gene sequences between exons 2 and 10 was performed on 11 Microminipigs, six pigs that were CD4 antibody reactive and five pigs that were CD4 antibody unreactive. DNA sequencing and analysis of the 11 PCR products identified three 
homozygous and three heterozygous allelic sequences from the six CD4 antibody reactive samples, and five homozygous allelic sequences from the five CD4 antibody unreactive samples. We aligned the eight homozygous allelic sequences against the swine CD4 reference sequence [GenBank: NM_001001908] and identified two distinct allelic nucleotide sequences between exons 2 and 10 of the CD4 genes that we classified as alleles CD4.A and CD4.B (Additional file 1). In comparison with the [GenBank: NM_001001908] sequence, the CD4.A [DDBJ: LC064059] and CD4.B [DDBJ: LC064060] alleles had 15 and 22 nucleotide substitutions between exon 2 and 10 regions, respectively (Table 3 ). Nucleotide sequences identical to CD4.A have not been found in GenBank, and so far appear to be unique to the Microminipigs. In contrast, the nucleotide sequences of $C D 4 . B$ were identical to that of the partial $C D 4.2$ sequence that reported only exons 3 and 4 in the CD4-undetectable NIH miniature swine [GenBank: X65630] [11].

In comparing the derived $\mathrm{CD} 4$ protein sequences with the swine $\mathrm{CD} 4$ amino-acid reference sequence [GenBank: NP_001001908], the CD4.A and CD4.B protein sequences had seven and 15 amino-acid substitutions, respectively, in the regions of exons 2 to 10 (Fig. 1, Table 4). In CD4.A, there was one amino-acid substitution in three of the four extracellular domains as well as in the joining regions 1 and 4, and two amino-acid substitutions in the transmembrane domain. In CD4.B, there were ten amino-acid substitutions in domain 1 , one in domain 3 , one each in joining regions 3 and 4 , and two in the transmembrane domain, some of which may change the polarity or charge of the amino-acid side chains. There was no amino-acid substitution in the cytoplasmic region of either CD4.A or CD4.B.

Three CD4 genotypes in Microminipig herd were assigned as CD4.AA, $A B$, and $B B$ by the PCR-RFLP method using $B s e$ RI (Fig. 2). The restriction enzyme patterns of CD4.AA, $A B$ and $B B$ showed a single band (366 bp), three bands (366, 260, and $106 \mathrm{bp}$ ), and two bands (260 and $106 \mathrm{bp}$ ), respectively. The matings of 17 pairs of heterozygous parents revealed that the inheritance pattern of CD4 genotypes was autosomal (Table 5). As shown with the flow cytometry results in Table 6, PBMCs with CD4.AA and $A B$ reacted with the antibody clone 74-12-4. In contrast,

Table 3 The number of nucleotide substitutions in CD4.A and CD4.B CDS compared to [GenBank: NM_00100908]

\begin{tabular}{lccccccccccc}
\hline Allele & $\begin{array}{l}\text { Exon } \\
\text { The number of nucleotide }\end{array}$ & 3 & 4 & 5 & 6 & 7 & 8 & 9 & 10 \\
\hline CD4.A & 0 & 1 & 1 & 2 & 3 & 3 & 5 & 1 & 0 \\
CD4.B & 0 & 12 & 1 & 1 & 3 & 0 & 5 & 1 & 0 \\
\hline
\end{tabular}

PBMCs with $C D 4 . B B$ were unreactive with the antibody. The MFI of $C D 4 . A B$ was approximately half the intensity of CD4.AA, even though the percentage of $\mathrm{CD} 4+$ cells in PBMCs were not different between the two CD4 alleles (Fig. 3).

CD4 gene expression was analyzed by RFLP using the RT-PCR sequence products that were amplified between exons 1 and 4 (Fig. 4a) or between exons 1 and 5 (Fig. 4b). RFLP distinguished the genotypes $C D 4 . A A, C D 4 . B B$ and $C D 4 . A B$ in both cases. In Fig. $4 \mathrm{a}$, the RT-PCR products were detected as a single 400 bp-band by electrophoresis. After BseRI digestion, the single band remained in $C D 4 A A$, whereas two digested bands of $303 \mathrm{bp}$ and $97 \mathrm{bp}$ were obtained in CD4.BB. Combinatorial band patterns of $C D 4 . A A$ and $C D 4 . B B$ were observed in $C D 4 . A / B$. In Fig. $4 \mathrm{~b}$, the undigested RT-PCR products were detected as a single 595 bp band. After the BseRI digestion, the single 595 bp band remained in CD4.AA, whereas two digested bands of $300 \mathrm{bp}$ and $295 \mathrm{bp}$ were obtained in $C D 4 . B B$, and the combinatorial band patterns of $C D 4 . A A$ and $C D 4 B B$ were observed in $C D 4 . A / B$. Consequently, these results suggested that PBMCs with heterozygous CD4 genotype coexpressed CD4.A and CD4.B alleles at the mRNA level.

In validating the expression vector sequences, the insertion sequences of CD4.A-FLAG and CD4.B-FLAG were found to be identical to the genomic exon sequences described above (Additional file 1) except for the added FLAG sequence. Moreover, we also found a spliced form that lacked the CD4 exon 8 in both of the two CD4 alleles. These spliced forms with the exon 8 deficiency gave rise to a stop codon at the $\mathrm{N}$-terminus of transmembrane domain as a result of a frameshift from the beginning of the exon 8 region, whereas amino-acid sequences of the external domains in the spliced forms were identical to those of the CD4.A and CD4.B derived from the nucleotide sequencing using genomic DNA (Fig. 5). Therefore, we used the constructs with complete sequences of CD4-FLAG for expression in HeLa cells. These alternative spliced forms were submitted to DDBJ (http://www.ddbj.nig.ac.jp) as CD4.A exon 8 deficiency [DDBJ: LC064061] and CD4.B exon 8 deficiency [DDBJ: LC064062].

Figure 6 shows the transient expression of CD4FLAG without the exon 8 deficiency in HeLa cells. The CD4.A and FLAG proteins in CD4.A-FLAG were detected with the anti-pig CD4 antibody and antiFLAG antibody. In contrast, the CD4.B in CD4.BFLAG was unreactive with the anti-pig CD4 antibody even though FLAG was detected with the anti-FLAG antibody. These results show that we expressed the CD4.B protein in HeLa cells, but that we could not detect it with the anti-CD4 antibody. 


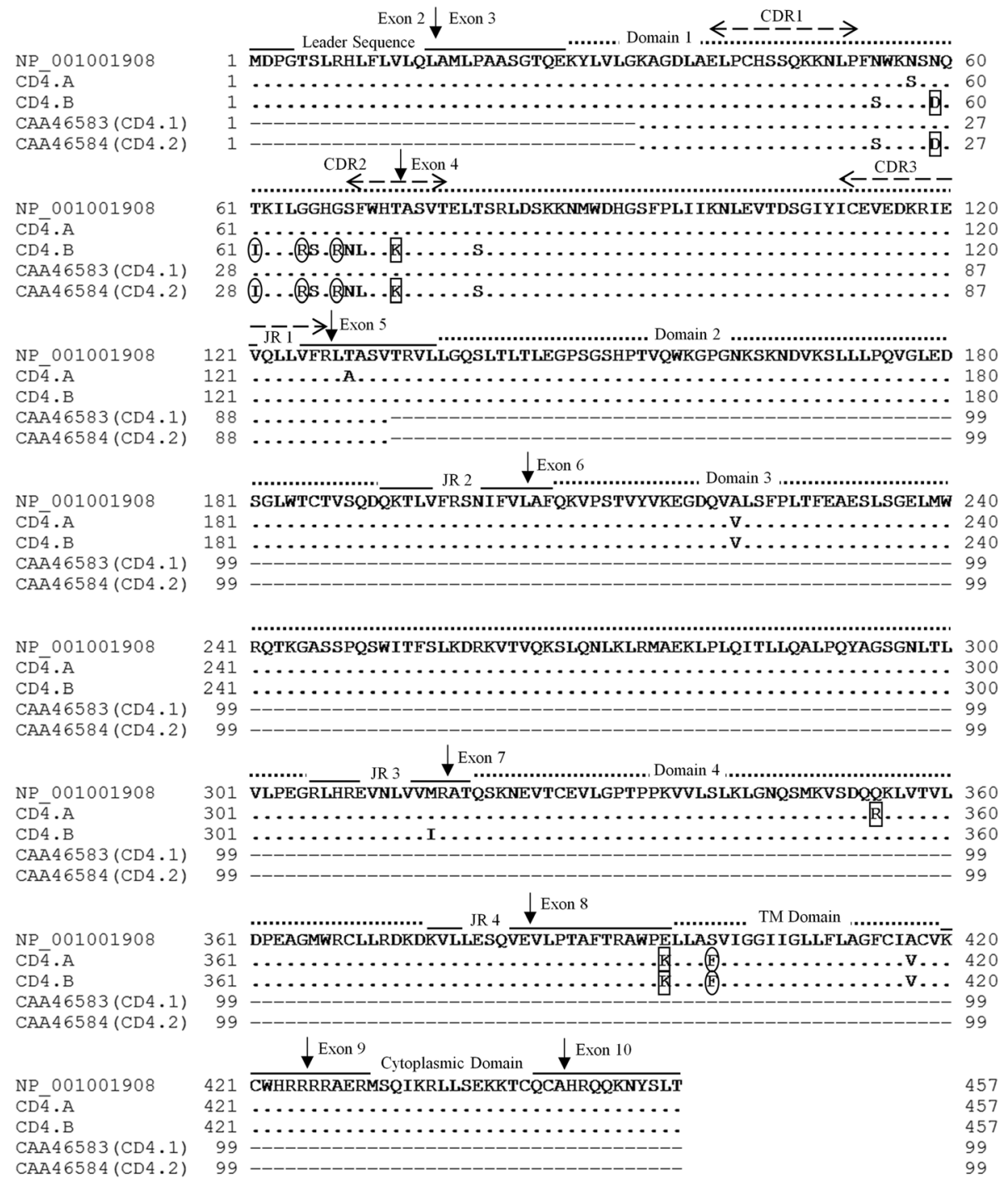

Fig. 1 Comparison of amino-acid sequences of porcine CD4 alleles. Deduced amino-acid sequences of CD4.A and CD4.B were compared with those of the swine CD4 reference sequence [GenBank: NP_001001908]. Amino-acid sequences of two CD4 alleles reported in NIH miniature swine [GenBank: CAA46583 (CD4.1), CAA46584 (CD4.2)] also were compared with CD4.A and CD4.B. (-) indicates gaps or absence of sequence corresponding to [GenBank: NP_001001908]. (.) indicates having identical sequence with that of [GenBank: NP_001001908]. Arrow indicates the putative boundary of each exon. Regions of leader sequence, four extracellular lg-like domains (Domain 1-4), joining region (JR 1-4), transmembrane (TM) domain, and cytoplasmic domain are also shown. $<--->$ indicates CDR1-3 like region in domain 1. An outlined circle indicates that the amino-acid side chain has changed the polarity in association with amino-acid substitution. An outlined square indicates that the amino-acid side chain has changed the charge in association with amino-acid substitution

Table 4 The number of amino-acid substitutions in CD4.A and CD4.B compared to [GenBank: NP_001001908]

\begin{tabular}{llllllllllllll}
\hline CD4 type & \multicolumn{1}{l}{ Regions of CD4 molecule } \\
\cline { 2 - 6 } & D 1 & JR 1 & D 2 & JR 2 & D 3 & JR 3 & D 4 & JR 4 & TM & CP \\
& \multicolumn{1}{l}{ The number of amino-acid substitutions } \\
\hline CD4.A & 1 & 1 & 0 & 0 & 1 & 0 & 1 & 1 & 2 & 0 \\
CD4.B & 10 & 0 & 0 & 0 & 1 & 1 & 0 & 1 & 2 & 0
\end{tabular}

D 1-4: domain 1-4; JR 1-4: joining region 1-4; TM transmembrane domain; CP cytoplasmic domain

\section{Discussion}

The CD4 exonic sequences of the Microminipig CD4 gene were analyzed using the DNA isolated from $\mathrm{CD} 4+$ cells that were CD4-reactive and non-reactive to the 74-12-4 antibody, and two corresponding alleles, CD4.A and $C D 4 . B$, respectively, were identified. Although the CD4 gene is thought to be highly conserved, CD4 polymorphisms were reported previously in human, bovine, and ovine [15-17]. Also, two CD4 partial allelic sequences were reported in NIH miniature swine (CD4.1: X65629; 


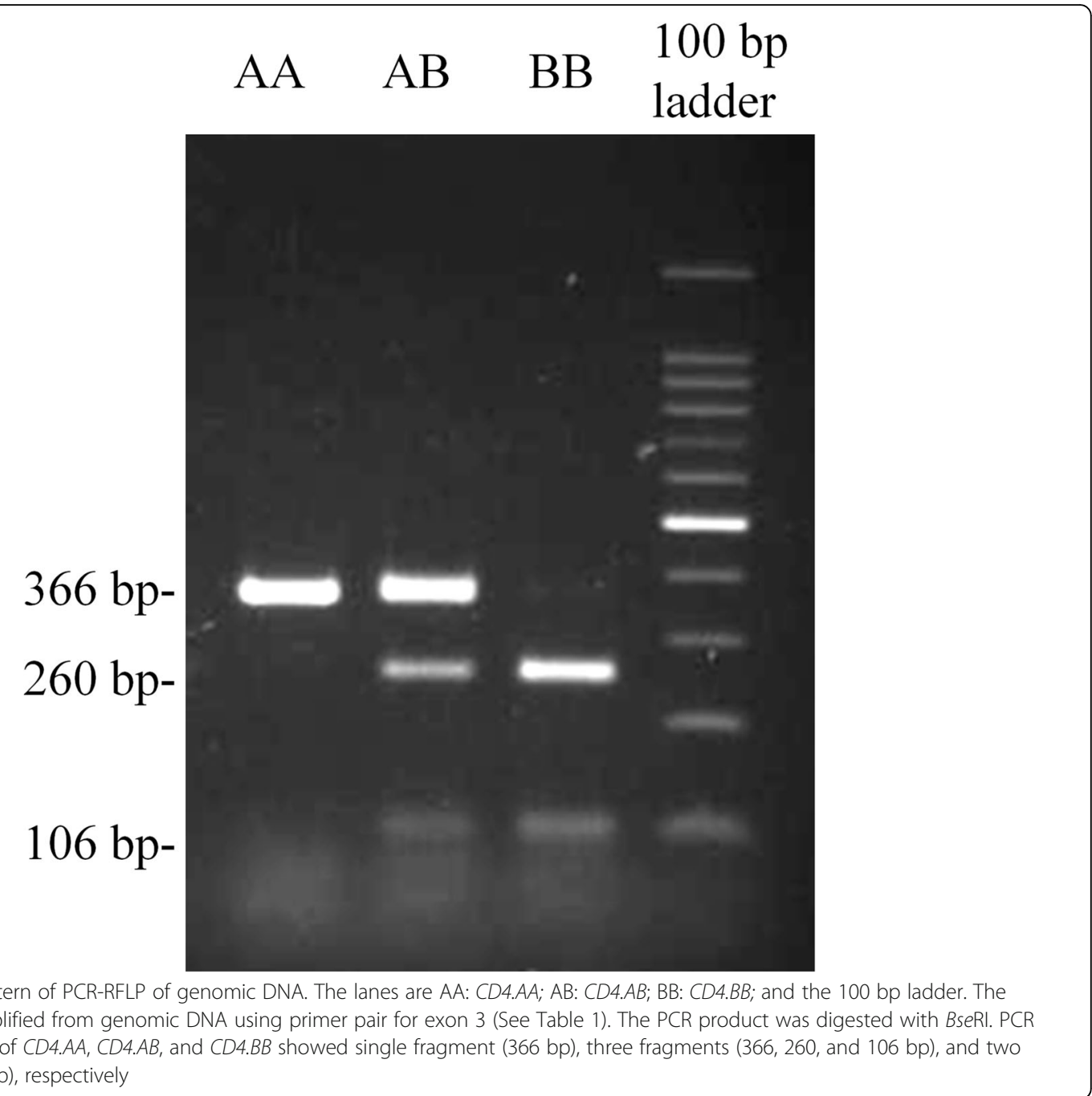

CD4.2: X65630) [11]. The nucleotide sequences of exons 3 and 4 of $C D 4 . B$ in Microminipigs were identical to those of CD4.2 in NIH miniature swine. We could not conclude that $C D 4 . B$ is identical to the complete CD4 gene sequence of NIH miniature swine because only the nucleotides of exons 3 and 4 were sequenced for CD4.2.

Table 5 CD4 genotypes of piglets delivered from the matings of CD4 heterozygous pigs

\begin{tabular}{|c|c|c|c|c|}
\hline \multirow{2}{*}{$\begin{array}{l}\text { CD4 } \\
\text { genotype }\end{array}$} & \multicolumn{2}{|c|}{ The number of piglets } & \multicolumn{2}{|c|}{ Theoretical value } \\
\hline & Male & Female & Male & Female \\
\hline$A A$ & 10 & 9 & 8 & 8 \\
\hline$A B$ & 16 & 13 & 16 & 16 \\
\hline$B B$ & 9 & 7 & 8 & 8 \\
\hline Total & 35 & 29 & 32 & 32 \\
\hline
\end{tabular}

The hereditary pattern corresponds to the theoretical value on the basis of autosomal heredity in heterozygous by analysis of chi-squared test $(P>0.05)$. Hence, the hereditary pattern of CD4 genotypes is autosomal in mode
However, the Microminipig breed is the result of crosses with western breeds, but it has no consanguinity with the $\mathrm{NIH}$ miniature swine [7]. Although the CD4.B of Microminipigs and $C D 4.2$ of $\mathrm{NIH}$ miniature swine might have a co-ancestor, the origins of $C D 4 . B$ and CD4.2 remain uncertain.

In comparing the amino-acid sequence alignments of CD4.A and CD4.B with the swine CD4 amino-acid reference sequence [GenBank: NP_001001908], we identified one amino-acid substitution in domain 4 of the extracellular region of CD4.A that involve alterations in the charge of the amino-acid side chain, and consequently a structural change in this domain of CD4. Domains 3 and 4 of CD4 play an important role in interacting with the $\mathrm{T}$ cell receptor-CD3 complex to influence signal transduction in T cell activation and function [18]. Therefore, the amino-acid substitution on domain 4 of CD4.A might affect signal transduction and $\mathrm{T}$ cell function during the interaction of $\mathrm{CD} 4$ with the $\mathrm{T}$ cell receptor-CD3 
Table 6 The relationship between CD4 genotype and affinity to anti-pig CD4 antibody

\begin{tabular}{llll}
\hline CD4 genotype & Number & CD4 reactivity & Number \\
\hline$A A$ & 24 & Reactive & 95 \\
$A B$ & 71 & & \\
$B B$ & 48 & Non-reactive & 48
\end{tabular}

CD4 genotype was assigned by PCR-RFLP method using BseRI. CD4 reactivity was veriified by flow cytometry. CD4+ cells in PBMCs could not be detected with the antibody when they carried the $C D 4 . B B$ genotype

complex. The five amino-acid substitutions observed in domain 1 of CD4.B also alter the polarity or charge of amino-acid side chains and might elicit a structural alteration in this domain of the CD4. The domain 1 of the $\mathrm{CD} 4$ combines with the monomorphic region of the $\mathrm{MHC}$ molecule in the presentation of antigenic determinants to activate selected lymphocytes [3, 4]. Moreover, the amino-acid substitutions in the region of domain 1 in CD4.B correspond to the CDR2-like region of CD4.2 in $\mathrm{NIH}$ miniature swine and the CDR2-like region of CD4 in humans that bind to MHC class II molecules and the HIV envelope glycoprotein gp120 [11]. No clinical abnormalities have been observed as yet with the $C D 4 . B$ genotype in Microminipigs, although the affinity of CD4.B to MHC class II might be different from that of CD4.A [9]. In this regard, the PCR-RFLP technique using $B s e$ RI has allowed us to identify the three $\mathrm{CD} 4$ genotypes $C D 4 . A A, A B$ and $B B$ that correlated with $C D 4$ reactivity to anti-pig CD4 antibodies. This simple CD4 genotyping method might be useful for selectively breeding CD4.A or CD4.B homozygous pigs and for developing association studies of immunity to infections and immunologically-related diseases.
Our PCR-RFLP study has demonstrated that both CD4.A and CD4.B are co-expressed in PBMC of heterozygous pigs. Moreover, the study on the expression of CD4-FLAG in HeLa cells confirmed that the amino-acid substitutions in CD4.B were associated with the loss of affinity to anti-CD4 antibody. On the other hand, the seven amino-acid substitutions observed within CD4.A reacted with the anti-CD4 antibody and one or other of them are likely to be linear or sequential epitopes recognized by the anti-CD4 antibody. Therefore, one or more of the ten amino-acid substitutions in domain 1 and/or the one amino-acid substitution in joining region 3 of CD4.B, which are not found in CD4.A, may have replaced the antigenic determinant and caused the lose of affinity for the anti-pig CD4 antibodies.

The detection of reduced levels of MFI in CD4 cells with $C D 4 . A B$ in the Microminipigs appears to be due to the anti-CD4 antibody reacting with CD4.A, but not with the CD4.B molecules, if both types of CD4 proteins are coexpressed on the surface of helper $\mathrm{T}$ cells. Moreover, in CD4-FLAG insertion sequencing, the $C D 4 . A$ and CD4.B alleles also had exon 8 deficiency forms that lacked the subsequent of transmembrane domain in the deduced amino-acid sequences. Thus, if the CD4 transcripts are without a transmembrane domain then the translated proteins might be secreted into the serum rather than be bound within the cellular membrane. The alternative spliced forms of CD4 CDS in swine have not been reported previously even though there are such variants registered in GenBank [GenBank: XM_005652591, GenBank: XM_005652592, GenBank: KC333254, GenBank: AY515293]. However, the lack of CD4 transmembrane region was reported for a mutant mouse model that secreted soluble CD4 without expression

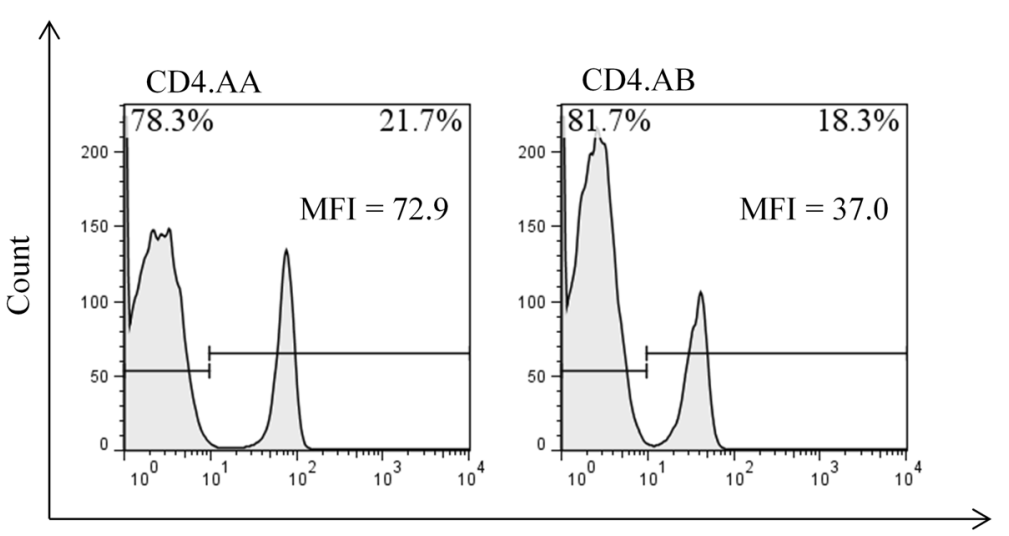

CD4 fluorescence intensity

Fig. 3 The percentage and MFI of CD4+ cells in PBMCs with CD4.AA and CD4.AB. CD4 genotypes in 2-month old Microminipigs assigned by PCRRFLP. PBMCs were stained with FITC-conjugated anti-pig CD4 antibody and assessed by flow cytometry as described in Materials and Methods. The MFI of CD4+ cells with CD4.AB was almost half of those with CD4.AA, even though there was no significant difference in the percentage of CD4+ cells between CD4.AA and CD4.AB 


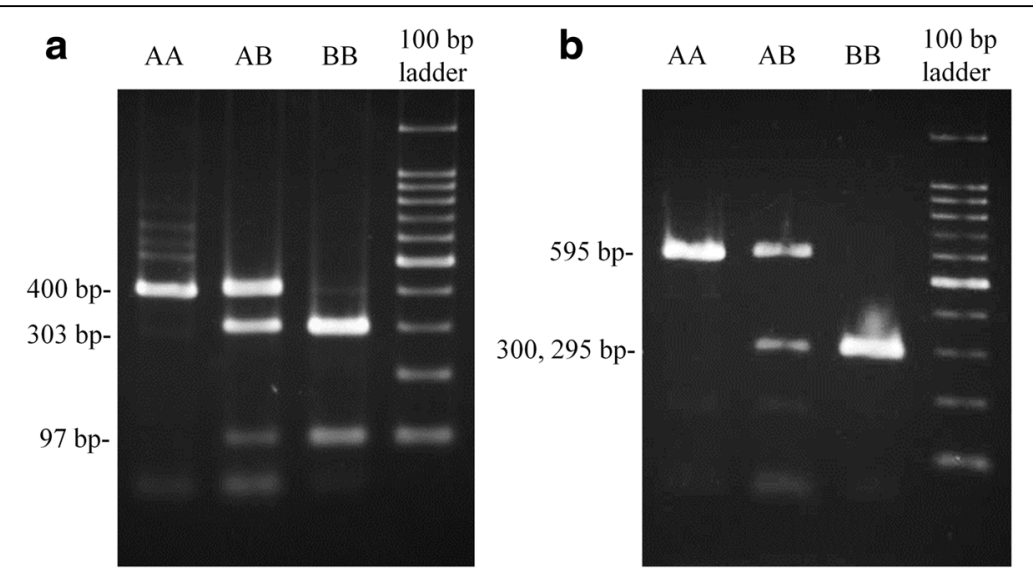

Fig. 4 Electrophoretic pattern of RT-PCR products after enzyme digestion with BseRI. The lanes are AA: CD4.AA; AB: CD4.AB; BB: CD4.BB; and the $100 \mathrm{bp}$ ladder. The $400 \mathrm{bp}$ (a) and $595 \mathrm{bp}$ (b) of the CD4 sequence were amplified from cDNA using primer sets shown in Table 2 and the amplified products were digested with BseRl. a After digestion with BseRI, CD4.AA, CD4.AB, and CD4.BB showed a 400 bp-fragment (400 bp), three fragments of 400, 303 and $97 \mathrm{bp}$, and two fragments of 303 and 97 bp, respectively. b After digestion with BseRl, CD4.AA, CD4.AB, and CD4.BB showed a 595 bp-fragment, three fragments of 595, 300 and 295 bp, and two fragments of 300 and 295 bp, respectively

of membrane-bound CD4 [19]. This mouse model was used to show that soluble CD4 impaired a delayed-type hypersensitivity response by inhibiting IFN- $\gamma$ production, and prohibited over-activation of CD4+ T cells by competitive inhibition of the binding of $\mathrm{CD} 4$ on the T-cell surface to MHC class II [20]. So, if the exon 8 deficiency forms are translated to the protein, the secreted CD4 might be also associated with prohibiting over-activation of CD4+ T cells in swine. Thus, further studies are needed to elucidate the significance of the expression of CD4 exon 8 deletions.

In NIH miniature swine, the functional differences of CD4 between CD4.1 and CD4.2 were investigated, but no differences were detected in antibody production against staphylococcal nuclease immunization and in the allogeneic mixed lymphocyte reaction [21]. In this regard, additional studies will be needed in Microminipigs to elucidate the functional significance or immunological importance of polymorphisms of the CD4 gene including the possible alternative spliced forms of the expressed gene. Because the CD4 molecule interacts with the MHC class II complex in antigen recognition $[3,4]$ the polymorphisms of both $\mathrm{CD} 4$ and MHC class II will need to be considered in future studies. The Microminipigs with defined SLA haplotypes [8] could be a useful animal model for further research on the interaction between $\mathrm{CD} 4$ allomorphs and MHC molecules in disease, infection and transplantation studies.

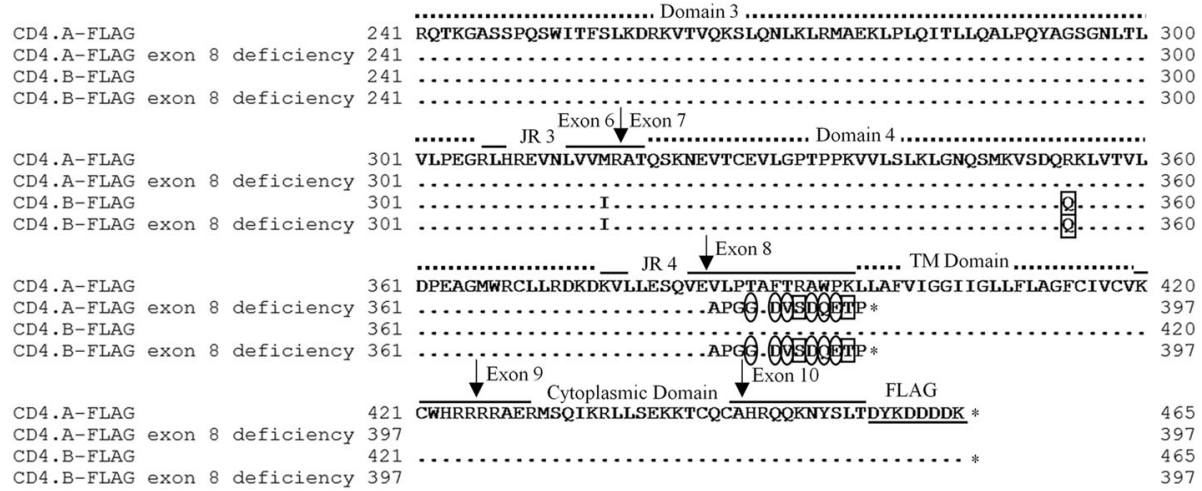

Fig. 5 Alignment of amino-acid sequences of CD4.A-FLAG and CD4.B-FLAG and their exon 8 deficiency forms. (.) indicates having identical sequence to CD4.A-FLAG. Arrow indicates the putative boundary of each exon. $\left(^{*}\right)$ indicates the stop codon. The regions of two extracellular lg-like domains (Domain 3, 4), joining region (JR 3, 4), transmembrane (TM) domain, cytoplasmic domain and FLAG are also shown. Exon 8 deficiency forms gave rise to a stop codon at the $\mathrm{N}$-terminus of TM domain as a result of a frameshift from the beginning of the exon 8 region, even though amino-acid sequences of their external CD4 domains in the spliced forms were identical to those of CD4.A-FLAG and CD4.B-FLAG. Thus, we used the constructs with complete sequences of CD4.A-FLAG and CD4.B-FLAG for the expression study in HeLa cells 


\section{CD4.A-FLAG}
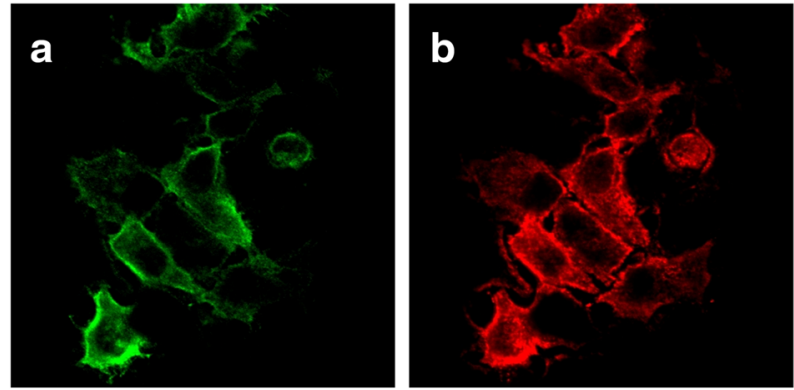

CD4.B-FLAG
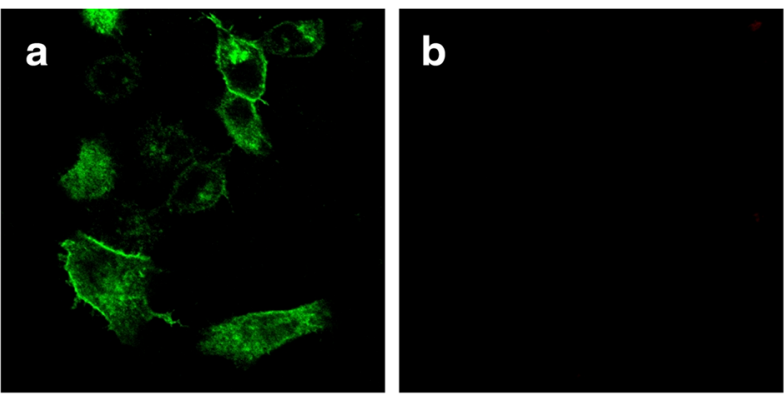

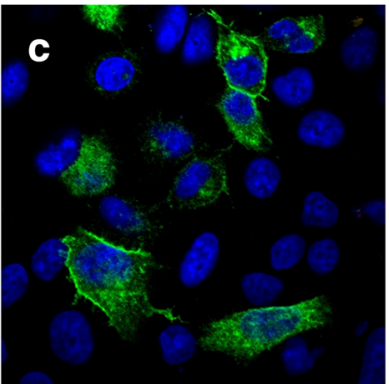

Fig. 6 Expression of the CD4-FLAG vectors in HeLa cells. We expressed only the constructs CD4-FLAG with complete sequences in HeLa cells as described in Fig. 5. a Cells stained with rabbit anti-FLAG antibody followed by Alexa Fluor 488 goat anti-rabbit lgG antibody. b Cells stained with PE conjugated mouse anti-pig CD4 antibody. c Cells stained with the overlay of three fluorophore signals; anti-FLAG antibody, anti-pig CD4 antibody, and Hoechst 33342

\section{Conclusions}

Two CD4 alleles, named as CD4.A and CD4.B, were identified in Microminipigs, and these two alleles were found to express an exon 8 deficiency, indicating the potential for alternative spliced forms. The loss of reactivity of antigenic epitopes to an anti-CD4 antibody probably resulted because of amino-acid substitutions in CD4.B. These CD4 polymorphisms could be genotyped and identified simply by the PCR-RFLP technique using BseRI in Microminipigs.

\section{Additional file}

Additional file 1: Alignment of CD4 CDS based on swine CD4 reference sequence [GenBank: NM_00100908]. Nucleotide sequences of CD4.A and CD4.B were aligned based on swine CD4 reference sequence [GenBank: NM_00100908], and two CD4 alleles reported in NIH miniature swine were also aligned [GenBank: X65629 (CD4.1), GenBank: X65630 (CD4.2)]. (-) indicates having no sequence corresponding to [GenBank: NM_00100908]. (.) indicates having identical sequence with that of [GenBank: NM_00100908]. An arrow indicates an exon boundary. (TIF 1208 kb)
Availability of data and materials

Nucleotide sequences of CD4.A and CD4.B with swine CD4 reference sequence [GenBank: NM_00100908] and two CD4 alleles reported in NIH miniature swine [GenBank: X65629 (CD4.1), GenBank: X65630 (CD4.2)] were shown in the supplementary file. Other data supporting our findings is contained within the manuscript.

\section{Authors' contributions}

TM participated in performing sequencing of CD4 and flow cytometry, and certifying the expression of CD4 mRNA and molecule, collected samples, analyzed data, and drafted the manuscript. NN, ST, and KOA participated in experimental design, performed sequencing of CD4 and certifying the expression mRNA, and drafted the manuscript. MT participated in sample collection and performed flow cytometry. NI contributed collecting samples and extracting DNA. KY drafted the manuscript. MK, ST, and YA conceived the expression study of CD4 molecule, participated in experimental design, coordinated experiments. YK contributed flow cytometric analysis and drafted the manuscript. JK drafted the manuscript. AA contributed sequencing analysis and drafted the manuscript. HK participated in experimental design, coordinated experiments, and drafted the manuscript. All authors read and approved the final manuscript.

\section{Competing interests}

The authors declare that they have no competing interests.

\section{Consent for publication}

Not applicable.

Ethics approval and consent to participate

This study protocol was approved by the institutional Animal Experiment Committee of Gifu University (approval no. 09045).

\section{Funding}

The authors received no financial support for this study.
We wish to express our appreciation to assistance of Fuji Micra Inc. for sample collection. 


\section{Author details}

United Graduate School of Veterinary Sciences, Gifu University, Gifu 501-1193, Japan. ${ }^{2}$ Department of Veterinary Medicine, Faculty of Applied Biological Sciences, Gifu University, 1-1 Yanagido, Gifu 501-1193, Japan. ${ }^{3}$ Viral Infectious Diseases Unit, RIKEN, Wako 351-0198, Japan. ${ }^{4}$ Department of Molecular Life Science, Division of Basic Medical Science and Molecular Medicine, Tokai University School of Medicine, Isehara 259-1193, Japan. ${ }^{5}$ School of Psychiatry and Clinical Neurosciences, The University of Western Australia, Crawley, WA 6009, Australia.

Received: 25 September 2015 Accepted: 4 October 2016 Published online: 07 October 2016

\section{References}

1. Kwong PD, Ryu SE, Hendrickson WA, Axel R, Sweet RM, FolenaWasserman G, et al. Molecular characteristics of recombinant human CD4 as deduced from polymorphic crystals. Proc Natl Acad Sci U S A. 1990;87:6423-7.

2. Maddon PJ, Molineaux SM, Maddon DE, Zimmerman KA, Godfrey M, Alt FW, et al. Structure and expression of the human and mouse T4 genes. Proc Natl Acad Sci U S A. 1987;84:9155-9.

3. König R, Huang LY, Germain RN. MHC class II interaction with CD4 mediated by a region analogous to the MHC class I binding site for CD8. Nature. 1992:356:796-8.

4. Miceli MC, Parnes JR. Role of CD4 and CD8 in T cell activation and differentiation. Adv Immunol. 1993;53:59-122.

5. Veillette A, Bookman MA, Horak EM, Samelson LE, Bolen JB. Signal transduction through the CD4 receptor involves the activation of the internal membrane tyrosine-protein kinase p56lck. Nature. 1989;338:257-9.

6. Holdorf AD, Lee KH, Burack WR, Allen PM, Shaw AS. Regulation of Lck activity by CD4 and CD28 in the immunological synapse. Nat Immunol. 2002:3:259-64.

7. Kaneko N, Itoh K, Sugiyama A, Izumi Y. Microminipig, a non-rodent experimental animal optimized for life science research: preface. J Pharmacol Sci. 2011;115:112-4.

8. Ando A, Imaeda N, Ohshima S, Miyamoto A, Kaneko N, Takasu M, et al. Characterization of swine leukocyte antigen alleles and haplotypes on a novel miniature pig line, Microminipig. Anim Genet. 2014;45:791-8.

9. Matsubara T, Nishii N, Takashima S, Takasu M, Imaeda N, Aiki-Oshimo K, et al. Identification of a CD4 variant in Microminipigs not detectable with available anti-CD4 monoclonal antibodies. Vet Immunol Immunopathol. 2015;168:176-83.

10. Sundt 3rd TM, LeGuern C, Smith CV, Sachs DH. Identification of a CD4 polymorphism in swine. Transplant Proc. 1991;23:419-20.

11. Gustafsson K, Germana S, Sundt 3rd TM, Sachs DH, LeGuern C. Extensive allelic polymorphism in the CDR2-like region of the miniature swine CD4 molecule. J Immunol. 1993;151:1365-70.

12. Pelchen-Matthews A, Armes JE, Marsh M. Internalization and recycling of CD4 transfected into HeLa and NIH3T3 cells. EMBO J. 1989;8:3641-9.

13. Maddon PJ, Dalgleish AG, McDougal JS, Clapham PR, Weiss RA, Axel R. Human immunodeficiency virus infection and syncytium formation in HeLa cells expressing glycophospholipid-anchored CD4. J Virol. 1991;65: 3276-83.

14. Niwa H, Yamamura K, Miyazaki J. Efficient selection for high-expression transfectants with a novel eukaryotic vector. Gene. 1991;108:193-9.

15. Tokito S, Kishi S, Yamamoto R, Takenaka T, Nakauchi H. Single amino acid substitution in the V3 domain of CD4 is responsible for OKT4 epitope deficiency. Immunogenetics. 1991;34:208-10.

16. Morrison WI, Howard CJ, Hinson CJ, MacHugh ND, Sopp P. Identification of three distinct allelic forms of bovine CD4. Immunology. 1994;83:589-94.

17. Boscariol R, Pleasance J, Piedrafita DM, Raadsma HW, Spithill TW. Identification of two allelic forms of ovine CD4 exhibiting a Ser183/Pro183 polymorphism in the coding sequence of domain 3. Vet Immunol Immunopathol. 2006;113:305-12.

18. Vignali DA, Carson RT, Chang B, Mittler RS, Strominger JL. The two membrane proximal domains of CD4 interact with the T cell receptor. J Exp Med. 1996;183:2097-107.

19. Nagase H, Wang CR, Yoshimoto T, Sugishita C, Shiroishi T, Matsuzawa A, et al. Novel mutant mice secreting soluble CD4 without expression of membranebound CD4. Eur J Immunol. 1998;28:403-12.
20. Wang CR, Hino A, Yoshimoto T, Nagase H, Kato T, Hirokawa K, et al. Impaired delayed-type hypersensitivity response in mutant mice secreting soluble CD4 without expression of membrane-bound CD4. Immunology. 2000;100:309-16.

21. Sundt 3rd TM, LeGuern C, Germana S, Smith CV, Nakajima K, Lunney JK, et al. Characterization of a polymorphism of CD4 in miniature swine. J Immunol. 1992;148:3195-201.

\section{Submit your next manuscript to BioMed Central and we will help you at every step:}

- We accept pre-submission inquiries

- Our selector tool helps you to find the most relevant journal

- We provide round the clock customer support

- Convenient online submission

- Thorough peer review

- Inclusion in PubMed and all major indexing services

- Maximum visibility for your research

Submit your manuscript at www.biomedcentral.com/submit
Biomed Central 